\title{
La competición en el diseño de las tareas de entrenamiento y la competitividad en la formación del jugador joven de fútbol
}

\section{David Falcón1, Román Nuviala Nuviala, Alejandro Moreno-Azze, José Luís Arjol Serrano}

\author{
'Dpto. Expresión Musical, Plástica y Corporal. Universidad de Zaragoza \\ Email: dfalcon@unizar.es
}

RESUMEN: El fútbol profesional es un deporte donde priman los resultados y donde la competitividad es un valor que se exige a los jugadores que quieren jugar a un máximo nivel. En esta investigación se estableció el perfil competitivo de los jugadores de un equipo juvenil perteneciente a la estructura de un Club de Fútbol profesional español mediante el cuestionario Competitividad-10 y durante cuatro meses del periodo competitivo se comprobó su relación con los resultados de los jugadores en las tareas de entrenamiento, susceptibles de ser diseñadas con estructura competitiva, y en la competición liguera. Además, se observó que, sin que el entrenador conociera estos datos, los jugadores con mejores resultados en los entrenamientos disfrutaban de más minutos en los partidos de liga constatando la relación entre la competitividad y el rendimiento deportivo, mejorando de esta forma el proceso formativo.

PALABRAS CLAVE: fútbol, resultado, rendimiento, entrenamiento

\section{Competition in the design of training drills and competitiveness in the training of young football players}

\begin{abstract}
Professional football is a results-oriented sport where competitiveness is a requirement for every single player who wants to excel at the highest level of competition. In this research, a competitive profile was established for the members of an U-18 team that belongs to a Spanish professional football club structure by means of the competitiveness-10 questionnaire. A competitiveness-results relationship was checked in a four-month competition timeframe in the area of training skills that were capable of being designed, or not, under the umbrella of a competitive structure, and during the league matches. Furthermore, it was observed that football players, who gave the best results during training sessions, were allowed to play more minutes in league matches, without the trainer being aware of the research results beforehand. This conclusion validates the relationship between competitiveness and high performance, improving the training process in this way.
\end{abstract}

KEY WORDS: soccer, result, training, high performance 


\section{INTRODUCCIÓN}

A la práctica deportiva se le atribuyen numerosas virtudes y valores como la amistad, la autoestima, la cooperación, la deportividad, y la competitividad, entendida ésta como auto superación personal (Valdemoros, 2010). Dentro de la Psicología del Deporte, existe la opinión compartida de que ciertos factores de personalidad están relacionados con la actividad física y el deporte (Allen, Greenless y Jones, 2013; De Francisco, Garcés de los Fayos y Arce, 2014; García-Naveira y Ruiz, 2013a; 2013b; Ruiz y García-Naveira, 2013) siendo la competitividad una de esas variables importantes a estudiar para comprender las características psicológicas asociadas a los deportistas de rendimiento (García-Naveira y Díaz, 2010; Gould, Dieffenbach, y Moffatt, 2002; García-Naveira y Remor, 2011).

Según las líneas de trabajo en este ámbito de diversos autores (e.g. GriffinPierson, 1990; Gill, Williams, Dowd y Beaudoin, 1996; Houston, Harris, Moore, Brummett y Kmetani, 2005) el estudio de la competitividad se encuadra dentro del ámbito de la investigación sobre la motivación de logro. En este marco la competitividad es entendida como una característica personal que influye sobre la conducta humana en diferentes dimensiones de la vida, como la social, la laboral o la deportiva, y que se refiere al "deseo de ganar en situaciones interpersonales" (Helmreich y Spence, 1978, p. 4), o disfrutar con la competición interpersonal y el deseo de ser mejor que otros (Spence y Helmreich, 1983). La competitividad, según Martens (1975) es una disposición para el esfuerzo con el objetivo de satisfacer un estándar de excelencia en las situaciones donde se realizan comparaciones en la presencia de evaluadores externos. Por tanto, la competitividad se constituye en una conducta de logro en un contexto competitivo, donde la evaluación social es el componente clave (Remor, 2007).

\subsection{Competitividad y nivel deportivo}

Diferentes estudios concluyen que los deportistas de mayor nivel deportivo son más competitivos que los de menor nivel, y las personas más competitivas obtienen mayor rendimiento deportivo que las menos competitivas (García-Naveira, RuizBarquín y Ortín, 2015). En el caso concreto del fútbol, García-Naveira y Remor (2011) concluyeron que el rasgo competitividad y la dimensión motivación de éxito en jugadores de fútbol de rendimiento de 14-24 años presentaban una relación positiva con el rendimiento deportivo, mientras la relación era negativa con la dimensión motivación para evitar el fracaso. En estudios anteriores, García-Naveira y Remor (2008) concluyeron también que los futbolistas de alto rendimiento entre los 20 y los 24 años obtienen mayores puntuaciones en la dimensión motivación de éxito y el rasgo de competitividad y menores puntuaciones en la dimensión motivación para evitar el fracaso que los deportistas amateurs. Estos autores concluyen que los deportistas que compiten en alto nivel han convivido dentro de un medio selectivo como es la competición, donde los deportistas más competentes y competitivos suelen ir consiguiendo éxitos deportivos y progresando en sus carreras.

\subsection{Competitividad y edad}

Según Martin y Ecklund (1994), la competitividad varía de forma curvilínea en función de la edad de los deportistas, presentando un aumento durante la niñez, la juventud y el inicio de la vida adulta y un descenso al acabar este periodo y adentrarse en la vejez. Algunos estudios revisados no apuntan una relación clara entre nivel de competitividad y edad de los deportistas. Beaudoin (2006) no obtiene relación entre la edad y la competitividad en jugadoras de fútbol entre los 18 y 45 años y Remor (2007) llegó a una conclusión similar en su estudio con deportistas universitarios de 19-32 años. 


\subsection{Competitividad y competición. Diseño de las tareas de entrenamiento}

La competición, es de significativa importancia para la motivación y formación de los jóvenes (Reverter, Mayolas, Adell y Plaza, 2009) El entrenador es el último responsable de escoger el tipo de tareas o de contenidos del entrenamiento, y cualquier tipo de tarea podemos plantearla para que finalice o no con ganadores y perdedores. Si introducimos y normativizamos los mecanismos necesarios para comparar el resultado de un participante o un grupo de participantes con respecto a otros, organizando a los jugadores en ganadores y perdedores habremos incluido la competición en el juego o en la tarea de entrenamiento (Duran, 2017). Una vez hecho esto, para que el jugador sea consciente del resultado, necesitaremos de un sistema de tanteo, que "es la red de aciertos, victorias o puntos conseguidos por los jugadores o los equipos de un juego deportivo, previstos explícitamente por el código de juego, tanto por lo que se refiere al desarrollo de todas las posibilidades como a la designación eventual de los ganadores y perdedores" (Parlebas, 2001, p. 421).

Si el final viene determinado por las reglas, el juego avanza inexorablemente hacia su propia conclusión y único propósito: determinar el vencedor de la contienda (Etxebeste, Del Barrio, Urdangarin, Usabiaga, y Oiarbide, 2014). Estos mismos autores denominan estos juegos como juegos con memoria de resultado o de competición y definen cuatro casos posibles que el entrenador puede elegir para terminar la tarea:

a) Sistema de clasificación. Todos los participantes se ordenan en función de una escala o clasificación: tiempo, distancia, puntuación u otros. Los concursos de habilidad entre los jugadores son buenos ejemplos de este tipo de tareas.

b) Conclusión a tiempo límite. El resultado del juego se obtiene al terminar un tiempo acordado. Es en ese preciso momento cuando se define al ganador (se admite el empate). Son un ejemplo los partidos reducidos donde el ganador es el que meta el mayor número de goles en unos pocos minutos.

c) a puntuación límite. El final viene determinado a la obtención de un resultado determinado (impide el empate). Esto ocurre; por ejemplo, cuando planteamos una tarea de fútbol tenis donde el que consiga un cierto número de puntos gana la partida

d) Puntación y duración límite. Este último caso hace referencia a los juegos deportivos que finalizan tras un tiempo o un resultado límite como ocurre en un partido con equipos a salidas donde un equipo vencerá o perderá al recibir una cantidad determinada de goles o al transcurrir un tiempo determinado (lo que ocurra primero).

e) Tareas donde los jugadores no actúan en dirección a un desenlace final, sino que sus participaciones se ven ligadas a una repetición de secuencias que pueden tener relación con los cambios de roles en el juego (Etxebeste et. al., 2014). En estos casos, la finalización del encuentro se produce a causas externas al juego (por cansancio, porque el entrenador decide cambiar de actividad, por acabar la hora del entrenamiento...) viéndose asociado todo el proceso de aprendizaje al proceso, a lo que sucede durante la partida, y no al resultado final.

Cualquier tarea o juego de entrenamiento con memoria de resultado ha sido registrado para la investigación, anotando los resultados individuales de cada jugador con el objetivo de comprobar si cuando un jugador se considera competitivo, y además un cuestionario así nos lo dice, es capaz de traducir esa competitividad en resultados victoriosos en las tareas y partidos. 


\section{MÉTODO}

\subsection{Participantes}

La muestra de deportistas del estudio estaba compuesta por 21 sujetos, hombres con una media de edad $\mathrm{M}=17.3$ años $\mathrm{DT}=.42$, pertenecientes a la estructura de un Club de Fútbol profesional español. Los jugadores realizan 4 sesiones de entrenamiento semanales de unos 120' de duración y compiten en categoría nacional juvenil.

\subsection{Variables e instrumentos}

El diseño utilizado en la investigación fue descriptivo. Las variables objeto de estudio fueron:

- C: Rasgo de competitividad del jugador.

- Me: Resultado de motivación al éxito del jugador.

- Maf: Resultado de motivación de alejamiento al fracaso.

- PPTE: Promedio de puntos conseguidos en las tareas de entrenamiento (valorando: 3-victoria, 1-empate y 0-derrota en cada tarea y dividiendo el total de puntos conseguidos por las tareas realizadas). En las tareas cuya finalización se establecía por sistema de clasificación, se dividía a los participantes en terciles, otorgando 3 puntos a los integrantes del primer tercil, 1 punto a los del tercil central y 0 puntos a los jugadores del tercer tercil.

- PPVPC: Puntuación ponderada de victorias en los partidos de competición (tomando el resultado parcial del partido durante el periodo de participación del jugador, multiplicando por los minutos de juego y dividiendo por los minutos de duración del partido)

- MIN: total de minutos de juego en partidos.

Se utilizó la Escala de Competitividad-10 de Remor (2007). Se trata de un cuestionario breve de auto informe con 10 cuestiones sobre las motivaciones asociadas a la competitividad deportiva (por ejemplo: Deseo ser el mejor cada vez que compito). El formato de respuesta es tipo Likert ( 1 = casi nunca; 2 = algunas veces; 3 = a menudo). La escala distingue un resultado de Motivación al éxito (Me) y Motivación de alejamiento al fracaso (Maf) obteniendo una puntuación independiente para ambas dimensiones. El indicador global del grado del rasgo de competitividad $(C)$ se calcula mediante la diferencia entre $\operatorname{Me}$ y $\operatorname{Mef}(C=M e-M e f)$. Cuando la diferencia entre las dos dimensiones es positiva, lo que indica un valor superior para la Me, la persona presenta tendencia a tener alta motivación para competir. Las propiedades psicométricas del cuestionario están descritas en Remor (2007), en el que se obtiene $=.66$ para la motivación al éxito $y=.66$ para la motivación a evitar el fracaso, la fiabilidad de ambas es aceptable

\subsection{Procedimiento}

Se administraron los cuestionarios (solicitando permiso a los propios deportistas y a la dirección deportiva del club) y se registraron todas las tareas de 47 sesiones de entrenamiento (294 tareas), los resultados de las tareas competitivas (93 tareas) y los minutos jugados y resultado parcial de cada jugador en los partidos de la competición liguera (13 partidos) durante cuatro meses correspondientes al periodo competitivo. 


\subsection{Análisis de datos}

Se utilizaron las siguientes técnicas de análisis de datos: Se realizó la prueba de normalidad Shapiro-Wilk dado que la muestra era inferior a 50 sujetos obteniendo valores de normalidad en las variables Maf $(z=.928 ; p=.162), \mathrm{C}(\mathrm{z}$ $=.914 ; p=.086)$, PPTE $(z=.950 ; p=.403)$ y MIN $(z=.956 ; p=.504)$ y de no normalidad en Me $(z=.821 ; p=.002)$ y PPVPC $(z=.750 ; p=.000)$. Posteriormente se aplicaron pruebas no paramétricas como el índice de correlación de Pearson para conocer la relación entre las variables.

Para la analizar los datos se utilizó el programa estadístico SPSS v. 21.0.

\section{RESULTADOS}

El resultado medio de los jugadores en el cuestionario arrojó datos elevados (M $=.83$; DT =,46). Durante los 4 meses de entrenamientos cada jugador obtuvo una media de 1,4 puntos por tarea. El jugador con mejor coeficiente obtuvo 1.76 puntos por tarea por los 1.10 del peor. La puntuación ponderada en los partidos obtuvo un valor medio de 53.15 puntos, siendo 83.3 el valor obtenido por el jugador con mejores resultados. Uno de los jugadores no disputó ningún minuto de la competición durante el periodo comprendido por la muestra.

Se analizó mediante el coeficiente de correlación de Pearson la relación entre las diferentes variables. Se aprecia una relación positiva entre el resultado obtenido en el cuestionario de competitividad y las victorias en las tareas de entrenamiento ( $r=$ .314) (figura 1) y los partidos ( $r=.351$ ) (figura 2).

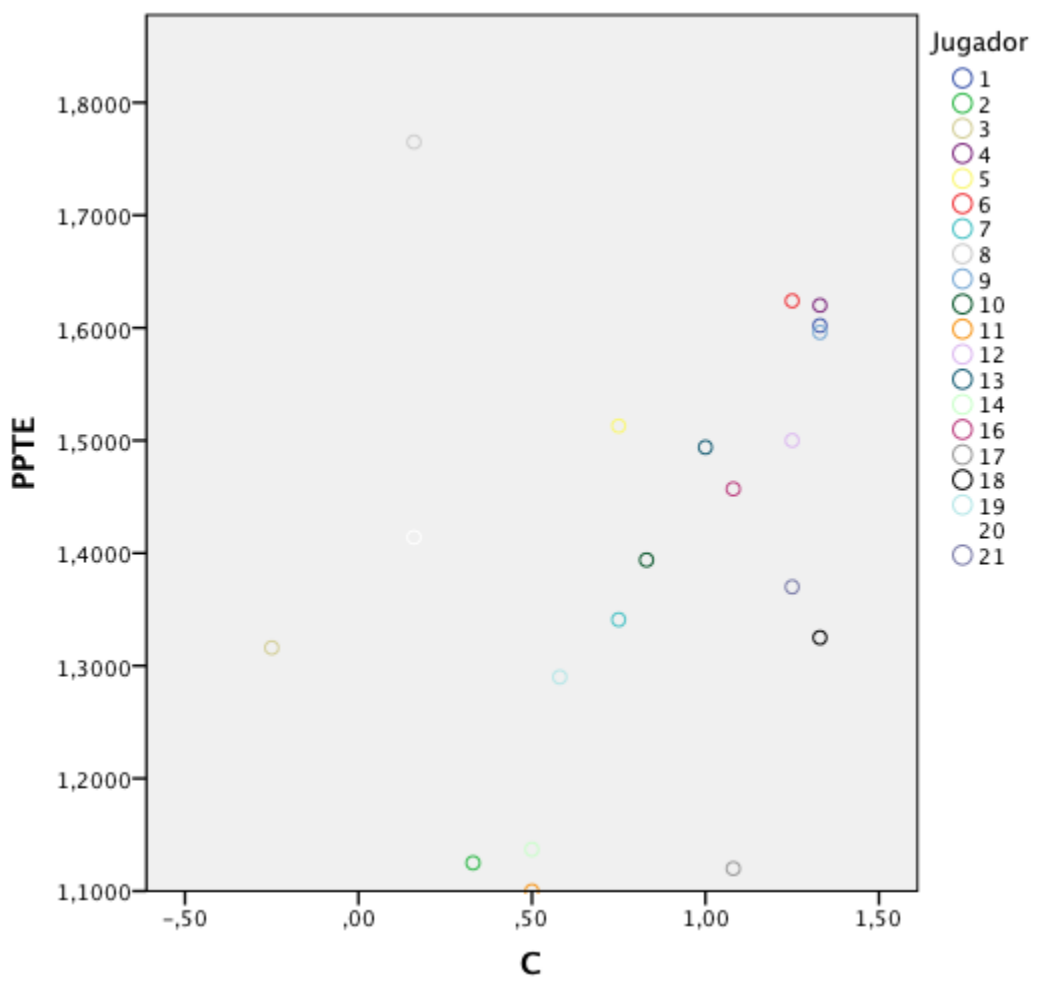

Figura 1. Gráfico de dispersión explicativo de la relación entre el Rasgo de Competitividad (C) y la puntuación obtenida en las tareas de entrenamiento (PPTE) 


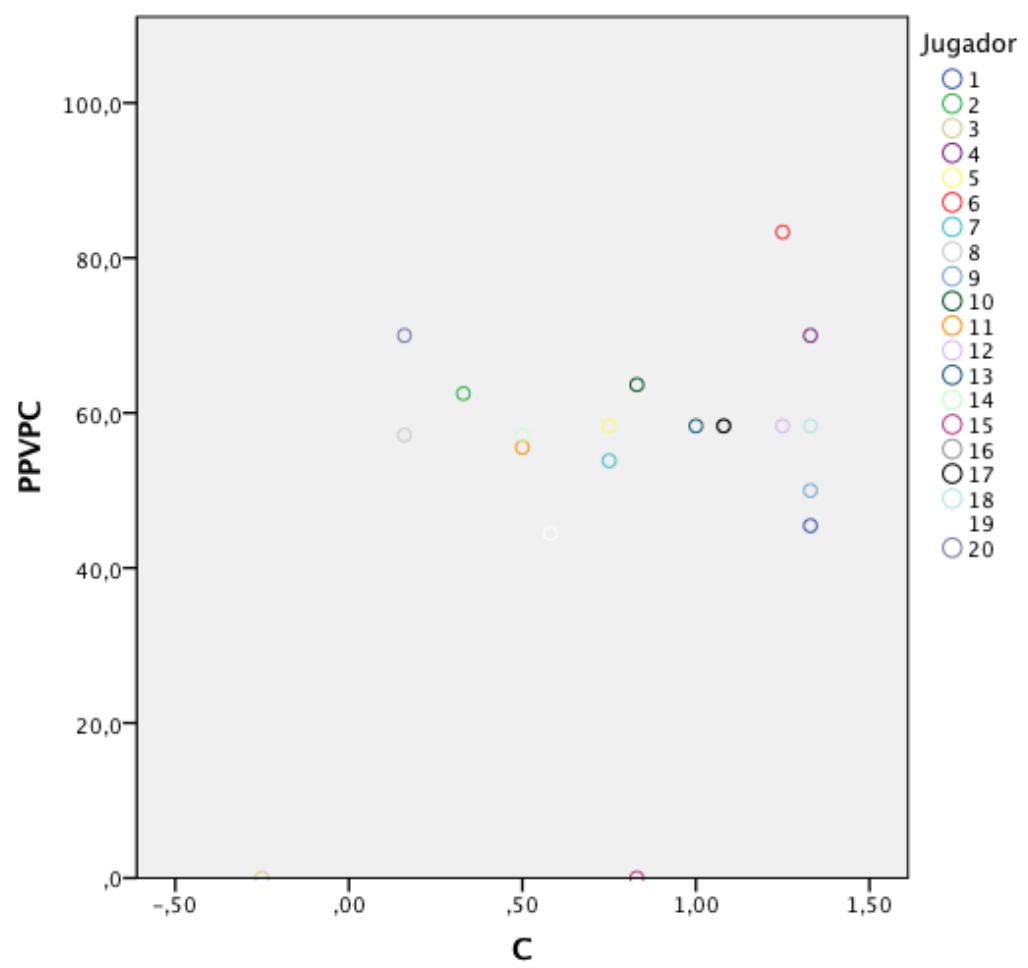

Figura 2. Gráfico de dispersión explicativo de la relación entre el Rasgo de Competitividad (C) y la puntuación ponderada obtenida en los partidos de competición (PPVPC)

La motivación al éxito también se ve relacionada directamente con las victorias en los partidos ( $r=.317$ ) (figura 3).

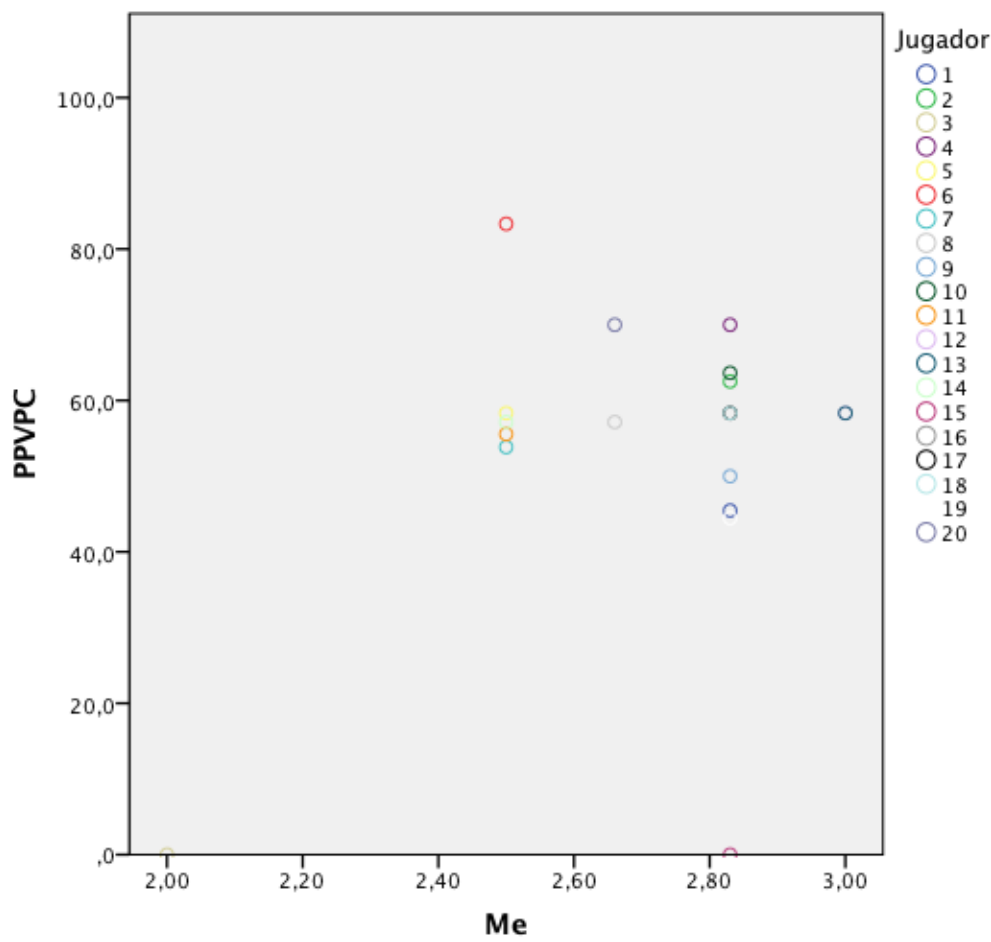

Figura 3. Gráfico de dispersión explicativo de la relación entre el Rasgo de Motivación para el éxito (Me) y la puntuación ponderada obtenida en los partidos de competición (PPVPC) 
Sin que el entrenador conociera el resultado del registro de las victorias en las tareas de entrenamiento, sí que se observa que los jugadores que mejores resultados han obtenido en las tareas son los que más minutos han disputado en los partidos ( $r=$ .316) (figura 4).

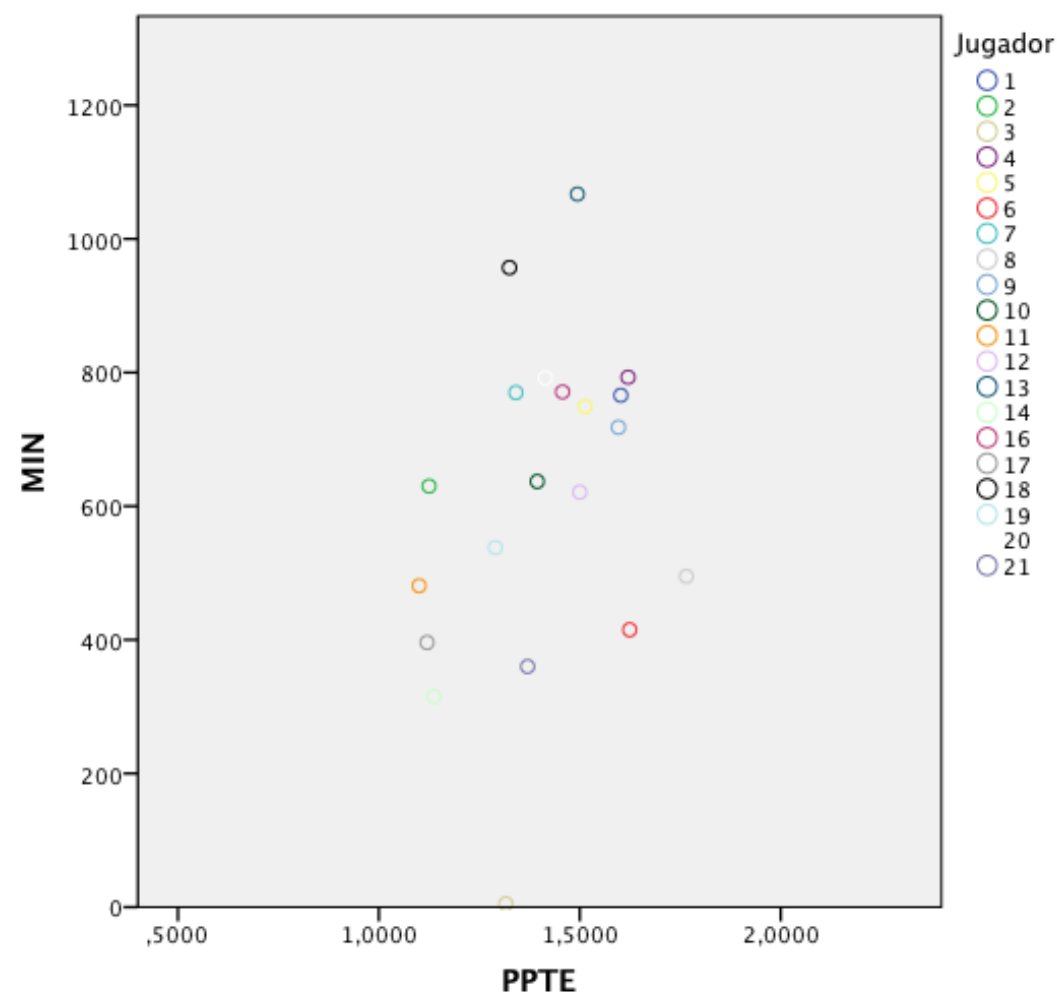

Figura 4. Gráfico de dispersión explicativo de la relación entre la puntuación ponderada obtenida en las tareas de entrenamiento (PPTE) y los minutos de juego en los partidos de competición (MIN)

\section{DISCUSIÓN}

Este estudio ha intentado tratar los aspectos relacionados a la competitividad del futbolista y su relación con el rendimiento, tomando como referencia el perfil competitivo del jugador desde un punto de vista psicológico y su transferencia a los resultados en el juego, tanto en entrenamientos como en partidos. El resultado medio de los jugadores en el cuestionario $(M=.83$; $\mathrm{DT}=.46)$ se acerca a los valores medios para deportistas de alto rendimiento en esa edad $(M=.90 ; D T=.52)$, según GarcíaNaveira et. al. (2015), por lo que podemos establecer como alto el nivel competitivo del grupo si lo comparamos con los valores de referencia de ese estudio. Diferentes trabajos citados anteriormente (García-Naveira y Remor, 2011; García-Naveira, RuizBarquín y Ortín, 2015) concluyen que las personas más competitivas obtienen mayor rendimiento deportivo que las no competitivas, lo que coincide con los resultados obtenidos en este estudio, donde los futbolistas con mayor puntuación en el test Competitividad-10 han obtenido mayores victorias en las tareas de entrenamientos y en los periodos de los partidos ligueros donde les ha tocado participar. García-Naveira y Remor (2011) concluyeron que el rasgo competitividad y la dimensión Me en jugadores de fútbol de rendimiento de 14-24 años se relacionaban positivamente con el rendimiento deportivo. Lorenzo-Gonzalez (1987) y Martín y Gill (1985) ya avanzaban la idea de que la motivación de logro es determinante en la competitividad deportiva. 
La relación obtenida en el estudio entre competitividad y victorias en las tareas concuerda con lo planteado por Weinberg y Gold (2003), que afirman que las personas altamente competitivas presentan tendencia a buscar situaciones de competición y están más motivadas para obtener éxito en ellas, si las comparamos con personas con bajo nivel de competitividad. Sin embargo, los autores advierten que el rasgo de competitividad aislado no predice adecuadamente como la persona responderá a una situación competitiva particular puesto que otras variables situacionales como la modalidad deportiva, los entrenadores, padres, 0 compañeros de equipo también ejercen una fuerte influencia sobre la conducta competitiva.

Los deportistas de alto nivel, sean profesionales o no, han convivido durante toda su formación en un medio selectivo como es la competición, donde los deportistas más competitivos y competentes van progresando y alcanzando éxitos deportivos mientras otros van quedando en el camino. En este sentido, según García-Naveira et. al. (2015), la conducta necesaria para superar a los rivales y conseguir el éxito en presencia de una alta evaluación social (público, familiares, jueces, técnicos y resto de jugadores) se traduce en el rasgo de competitividad. Los jugadores del estudio han obtenido mayores puntuaciones en las dimensiones $\mathrm{C}$ y $\mathrm{Me}$ que en la dimensión Maf al igual que en el estudio de García-Naveira y Remor (2008) lo que se relaciona positivamente con el rendimiento deportivo.

Diversos estudios destacan la capacidad de la competición en el deporte como elemento generador de motivación, compromiso, satisfacción y fuente de desarrollo personal (Cecchini, Fernández, González, y Arruza, 2008; Gutiérrez, Carratalá, Guzmán y Pablos, 2010; Sánchez-Oliva, Leo, Sánchez-Miguel, Amado, y García Calvo, 2012). En esta línea, hemos podido observar como la motivación de los jugadores al conocer que sus resultados se estaban registrando han provocado un aumento en la intensidad de los entrenamientos.

La necesidad de aproximarnos a lo que sucede en la competición no solo ha sido la base para la planificación y organización de los entrenamientos, sino que también ha servido como base informativa para apoyar la toma de decisiones de los entrenadores en la definición y ajuste del modelo de juego óptimo para la competición (Castelo, 1996; Teodorescu, 1984; Mombaerts, 1998). En el caso de este estudio, el entrenador, de forma intuitiva, ha utilizado a los jugadores que mejor se han desenvuelto en el contexto competitivo de los entrenamientos a la hora de afrontar la competición liguera, lo que hace que el trabajo realizado en esta investigación pueda convertirse en una herramienta útil para el entrenador a la hora de seleccionar a los jugadores adecuados para los partidos.

\section{CONCLUSIONES}

Los jugadores del equipo cumplen los valores de competitividad mostrados por deportistas de alto rendimiento en esa edad. Al introducir la competición en las tareas de entrenamiento aumentó la motivación de los jugadores y la intensidad de las sesiones. Los jugadores con mejor valoración en el test tienen mejores resultados en las tareas competitivas. Los jugadores de perfil más competitivo y con mejores resultados en las tareas de entrenamiento disfrutan de más minutos en la competición liguera. Se constata la relación entre la competitividad y el rendimiento deportivo. 


\section{REFERENCIAS}

Allen, M.S., Greenless, I., y Jones, M. (2013). Personality in sport: A comprehensive review. International Review of Sport and Exercise Psychology, 6, 184-208. doi:10.1080/1750984x.2013.769614

Beaudoin, C.M. (2006). Competitive orientations and sport motivation of professional women football players: An Internet survey. Journal of Sport Behavior, 29, 201212.

Castelo, J. (1996). A organizaçao do jogo. Lisboa: Edición do Autor.

Cecchini, J.A., González C., Carmona, A.M., y Contreras, O. (2004). Relaciones entre el clima motivacional, la orientación de meta, la motivación intrínseca, la autoconfianza, la ansiedad y el estado de ánimo en deportistas jóvenes. Psicothema, 16, 104-109.

De Francisco, C., Garcés de los Fayos, E.J., y Arce, C. (2014). Burnout en deportistas: Prevalencia del síndrome a través de dos medidas. Cuadernos de Psicología del Deporte, 14, 29-38.

Duran, C. (2017). La toma de conciencia emocional a través del juego deportivo en diferentes grupos de edad. Tesis doctoral no publicada, INEFC-Universitat de Lleida, Lleida.

Etxebeste, J., Del Barrio, S., Urdangarin, C., Usabiaga, O., y Oiarbide, A. (2014). Ganar, perder o no competir: la construcción temporal de las emociones en los juegos deportivos. Educatio Siglo XXI, 32 (1), 33-48.

García-Naveira, A., y Díaz. F. (2010). Relación entre optimismo/ pesimismo disposicional, rendimiento y edad en jugadores de fútbol de competición. Revista Iberoamericana de Psicología del Ejercicio y el Deporte, 5, 45-60.

García-Naveira, A., y Remor, E. (2011). Motivación de logro, indicadores de competitividad y rendimiento en un equipo de jugadores de fútbol de competición varones entre 14 y 24 años. Universitas Psychologica, 10, 477487.

García-Naveira, A., y Ruiz Barquín, R. (2013a). La personalidad del deportista: una revisión teórica desde la perspectiva de rasgos. Revista Internacional de Medicina y Ciencias de la Actividad Física y el Deporte, 13, 627-645.

García-Naveira, A., y Ruiz, R. (2013b). Diferencias de personalidad en entrenadores desde el modelo de Costa y McCRae. Cuadernos de Psicología del Deporte, $13,53-62$.

García-Naveira, A., Ruiz-Barquín, R., y Ortín, F. (2015). Optimismo y competitividad en jóvenes atletas de rendimiento. Revista Latinoamericana de Psicología, 47(2), 124-135.

Gill, D. L., Williams, L., Dowd, D. A. y Beaudoin, C. M. (1996). Competitive orientations and motives of adult sport and exercise participants. Journal of Sport Behavior, 19 (4), 307-318.

Gould, D., Dieffenbach, K., y Moffett, A. (2002). Psychological characteristics and their development in Olimpic champions. Journal of Applied Sport Psychology, 14, 
172-204. doi:10.1080/ 10413200290103482

Griffin-Pierson, S. (1990). The competitiveness questionnaire: A measure of two components of competitiveness. Measurement and Evaluation in Counseling and Development, 23 (3), 108-115.

Gutiérrez, M., Carratalá, V., Guzmán, J. F., y Pablos, C. (2010). Objetivos y manifestación de valores sociales y personales en el deporte juvenil según deportistas, padres, entrenadores y gestores. Apunts. Educación Física y Deportes, 101, 57-65.

Helmreich, R. L. y Spence, J. T. (1978). Work and Family Orientation Questionnaire: an objective instrument to assess components of achieving motivation and attitudes toward family and career. JSAS Catalogue of Selected Documents in Psychology, 8 (2), 35.

Houston, J. M., Harris, P. B., Moore, R., Brummett, R. y Kmetani, H. (2005). Competitiveness among japanese, chinese, and american undergraduate students. Psychological Reports, 97 (1), 205-212.

Martens, R. (1975). Social Psychology of Sport. New York: Harper \& Row.

Martin, J., y Ecklund, R. (1994). The relationships among competitiveness, age and ability in distance runners. Journal of Sport Behavior, 17, 258-266.

Mombaerts, E. (1998). Fútbol, entrenamiento y rendimiento colectivo. Barcelona: Hispano Europea.

Naveira, A., y Remor, E. (2008). Diferencias en competitividad en función del nivel competitivo en jugadores de fútbol adultos. XI Congreso Nacional de Psicología de la Actividad Física y el Deporte, XI Congreso Andaluz y III Iberoamericano. Sevilla, España.

Parlebas, P. (2001). Juegos, deporte y sociedad: Léxico de praxiología motriz. Barcelona: Paidotribo.

Remor, E. (2007). Propuesta de un cuestionario breve para la evaluación de la competitividad en el ámbito deportivo: Competitividad-10. Revista de Psicología del Deporte, 16, 167-183.

Reverter, J., Mayolas, C., Adell L. y Plaza, D. (2009). La competición deportiva como medio de enseñanza en los centros educativos de primaria. Retos: Nuevas tendencias en educación física, deporte y recreación, 16, 5-9.

Ruiz-Barquín, R., y García-Naveira, A. (2013). Personalidad, edad y rendimiento deportivo en jugadores de fútbol desde el modelo de Costa y McCrae. Anales de psicología, 29, 642-655. doi: 10.6018/analesps.29.3.175771

Sánchez-Oliva, D.; Sánchez-Miguel, P.A.; Leo, F. M.; Amado, D. y García-Calvo, T. (2013). Desarrollo y validación de un cuestionario para analizar la percepción de comportamientos positivos en las clases de educación física. Cultura y Educación, 25(4), 495-507.

Spence, J. T. y Helmreich, R. L. (1983). Achievement related motives and behavior. En J. T. Spence (Ed.) Achievement and achievement motives: Psychological and sociological dimensions. (pp. 7-74). San Francisco, CA: Freeman. 
Teodorescu, L. (1984). Problemas de teoría y metodología nos desportos colectivos. Lisboa: Livros horizonte.

Valdemoros, M.A. (2010). Los valores en el ocio físico-deportivo. Análisis y propuestas educativas. Logroño: Universidad de La Rioja.

Weinberg, R. y Gould, D. (2007). Foundations of sport and exercise psychology. Human Kinetics. 